BULL. AUSTRAL. MATH. SOC.

VOL. 28 (1983), 161-174.

\title{
CLOSED STRUCTURES ON THE CATEGORY OF TOPOLOGICAL SPACES DETERMINED BY SYSTEMS OF FILTERS
}

\author{
Maria Cristina Pedicchio
}

\begin{abstract}
We give a characterization of monoidal closed structures, "determined by systems of filters" on the category of topological spaces and continuous maps. The method we use to introduce suitable topologies on the product set $X \times Y$ of spaces $X$ and $Y$, and on the set of all continuous maps from $X$ to $Y$, is essentially that of Wilker.
\end{abstract}

\section{Introduction}

The following paper deals with monoidal closed structures (Top, $-\square-,[-,-], I, r, 1, a$ ) on Top (in the sense of [6]).

Many authors drew their attention to the study of such structures, not only in these last years, but even before category theory became relevant (see for instance [1], [4], [2], [5]).

It is known [9], that any such structures must necessarily have, as underlying set of $I, X \square Y$ and $[Y, Z]$, the canonical one, that is, $\{*\}, X \times Y$ (product set) and $\operatorname{hom}(Y, Z)$ respectively. So, to construct monoidal closed structures on Top, it suffices to provide the product set and the set of continuous functions with suitable topologies and to study when such spaces are related by an exponential law.

In this direction, we follow the approach of wilker [11], in Received 23 May 1983.

Copyright Clearance Centre, Inc. Serial-fee code: 0004-9727/83 $\$ A 2.00+0.00$. 
topologizing the above-mentioned sets by families of filters (see $\S I$ ). In $\S 2$ we give a characterization of those particular systems of filters, that generate monoidal closed structures on Top.

All the examples given by Booth and Tillotson in [2], and by Greve in [7], are of this kind.

Furthermore they seem to be all the examples known in literature on the matter.

\section{Notation}

We shall always use the following notations: $X, Y, Z$ denote topological spaces; $X \times Y$ indicates the product set of $X$ and $Y$ while $\operatorname{hom}(X, Y)$ denotes the set of continuous functions from $X$ to $Y .\{\star\}$ stands for a singleton space and $S$ for a Sierpinski space, that is, a space consisting of two points 0,1 , with $\varnothing,\{1\},\{0,1\}$ as its open sets.

For any topological space $Y, 0 p Y$ denotes the set of open sets of $Y$ and $\Phi$ the natural identification of $0 p Y$ with $\operatorname{hom}(Y, S)$. $\Phi$ is defined by $\Phi(U)=\Phi_{U}, U \in O p Y$, where $\Phi_{U}: Y \rightarrow S$ is the characteristic map of $U\left(\Phi_{U}(y)=I\right.$ if $y \in U, \Phi_{U}(y)=0$ if $\left.y \notin U\right)$. If $X \otimes Y$ and $(Y, Z)$ are arbitrary topological spaces on the product set $X \times Y$ and the set $\operatorname{hom}(Y, Z)$, respectively, then, by an exponential law $\psi$, we mean the bijection

$$
\psi: \operatorname{hom}(X \otimes Y, Z) \rightarrow \operatorname{hom}(X,(Y, Z))
$$

defined by

$$
\begin{aligned}
\psi(f)(x)(y) & =f(x, y), \\
\psi^{-1}(g)(x, y) & =g(x)(y),
\end{aligned}
$$

for any $f: X \otimes Y \rightarrow Z$ and $g: X \rightarrow(Y, Z)$ continuous maps.

The symbol $(K)$ with $K \subseteq Y$ and $Y \in$ Top, denotes the filter of open sets generated by $K$, that is,

$$
\langle K\rangle=\{U, U \supseteq K \mid U \text { open set of } Y\} \subseteq 0 p Y .
$$


Let $Y$ be a topological space, and $F$ a family of filters $F$ on $O p Y$ (that is, each $F \in F$ is a subset of the set $O p Y$ ). It is possible to define a topology on $0 p Y$, by requiring these sets $F$ to form an open subbase. Op $Y$ with this topology will be denoted by $T Y$. (If $F$ is the empty family, then $T Y$ is an indiscrete topological space.)

DEFINITION 1.1. For any $Z \in$ Top, we denote by $[Y, Z]$ the set $\operatorname{hom}(Y, Z)$ with the initial topology with respect to all the functions of the form

$$
\lambda_{U}: \operatorname{hom}(Y, Z) \rightarrow T Y
$$

with $U \in 0 \mathrm{p} Z$ and $\lambda_{U}(f)=f^{-1}(U)$, for any $f \in \operatorname{hom}(Y, Z)$.

The family of all the sets $W(F, U)=\left\{f, f \in \operatorname{hom}(Y, Z) \cdot \mid f^{-1}(U) \in F\right\}$, $U \in 0 p Z, F \in F$, is an open subbase of $[Y, Z]$. It is easy to see that, defining $[Y, h]=\operatorname{hom}\left(I_{Y}, h\right):[Y, Z] \rightarrow\left[Y, Z^{\prime}\right]$, for any $h: Z \rightarrow Z^{\prime},[Y,-]$ becomes a functor Top $\rightarrow$ Top . Furthermore $T Y \cong[Y, S]$ by $\Phi$ and $\lambda_{U}=\left[Y, \Phi_{U}\right]$.

Consider now the product set $X \times Y$. For any $A \subseteq X \times Y$, and $x \in X$, we use the symbol $A_{x}$ to denote the set $\{y, y \in Y \mid(x, y) \in A\}$.

DEFINITION 1.2. A subset $A$ of $X \times Y$ is an F-open set if and only if

(i) $A_{x} \in O p Y$, for any $x \in X$,

(ii) $u: X \rightarrow T Y, u(x)=A_{x}$, is a continuous map.

A family $F$ of filters on $O p Y$ is called an adjoining system, in the sense of Wilker [11], if and only if the two following conditions are satisfied:

(i) each $F \in F$ is a compact filter (that is, if $U U_{i} \in F$, $i \in I, \quad U_{i} \in O p Y$, then there is a finite subset $J \subseteq I$ such that $\left.U U_{i} \in F, i \in J\right)$; 
(ii) for any pair $U_{1}, U_{2} \in O p Y$ and for any $F \in F$, if $U_{1} \cup U_{2} \in F$, then there are filters $F_{1}, F_{2}$, finite intersections of filters of $F$, such that $U_{1} \in F_{1}$, $U_{2} \in F_{2}$ and $F_{1} \cap F_{2} \subseteq F$

Wilker proved in [11, p. 272, Theorem 2] that the F-open sets are the open sets for a topology on $X \times Y$, if and only if $F$ is an adjoining system.

We denote this topological space by $X \square Y$, and by $-\square Y:$ Top $\rightarrow$ Top the associated functor (where, for any $h: X \rightarrow X^{\prime}$, $h \square Y=h \times I_{Y}: X \square Y \rightarrow X^{\prime} \square Y$ ).

LEMMA 1.3. If $X \otimes Y$ denotes an arbitrary topology on the product set $X \times Y$, then the exponential low

$$
\psi_{X, Y, Z}: \operatorname{hom}(X \otimes Y, Z) \rightarrow \operatorname{hom}(X,[Y, Z])
$$

is a bijection for any $X$ and $Z$ in Top, if and only if $\psi$ is a bijection for any $X$ in Top and $Z=S$.

Proof. If $Z=S$ and $\psi_{X, Y, S}: \operatorname{hom}(X \otimes Y, S) \rightarrow \operatorname{hom}(X, T Y)$ is a bijection (where $[Y, S]$ is identified with $T Y$ ), then the open sets of $X \otimes Y$ are exactly the F-open sets and $X \otimes Y=X \square Y$. It follows that the inclusion map $i:\{x\} \times Y \rightarrow X \square Y$ is continuous, for any $x \in X$.

To prove that $\psi_{X, Y, Z}$ is a bijection, for any $X$ and $Z$, it suffices to verify that, given $f: X \square Y \rightarrow Z, g: X \rightarrow[Y, Z], x \in X$, the maps $\psi(f): X \rightarrow[Y, Z], \psi(f)(x): Y \rightarrow Z$ and $\psi^{-1}(g): X \square Y \rightarrow Z$ are continuous. $\psi(f)(x)=f(x,-)$, by definition of $\psi$, and

$$
f(x,-)=f \cdot i_{x}: Y \cong\{x\} \times Y \rightarrow X \square Y \rightarrow Z
$$

for any $x \in X$, so it is continuous.

For the universal property of initial topology, $\psi(f): X \rightarrow[Y, Z]$ is continuous if and only if the composite

$$
X \underset{\psi(f)}{\longrightarrow}[Y, Z] \underset{\lambda_{U}}{\longrightarrow} T Y
$$


is continuous, for any $U \in O p Z$; being $\psi_{X, Y, S}$ a bijection, such composite is continuous if and only if

$$
\psi_{X, Y, S}^{-1}\left(\lambda_{U} \cdot \psi(f)\right): X \square Y \rightarrow S
$$

is continuous.

Since this map is the characteristic map $\Phi_{A}$, associated to the open set $A=f^{-1}(U) \in O p \times \square Y$, the result follows.

If $g: X \rightarrow[Y, Z]$, let $\tilde{g}$ denote the function $\psi^{-1}(g): X \square Y \rightarrow Z$. Since $g$ is continuous, the composite

$$
X \underset{g}{\longrightarrow}[Y, Z] \underset{\lambda_{U}}{\longrightarrow} T Y
$$

is continuous, for any $U \in O p Z$; applying $\psi_{X, Y, S}^{-1}$ we obtain a continuous map of $X \square Y$ in $S$, which is exactly the characteristic map associated to the set $\tilde{g}^{-1}(U)$. Since $\tilde{g}^{-1}(U)$ is an open set for any $U \in O p Z$, the continuity of $\tilde{g}$ follows.

PROPOSITION 1.4. There is an adjunction $(-\square Y,[Y,-], \psi)$ if and only if $F$ is an adjoining system for $Y$.

Proof. This is a consequence of [11, p. 272, Theorem 2] and of Lemma 1.3, where $\psi$ is - of course - natural in $X$ and $Z$.

Suppose now we have an adjoining system of filters, $F(Y)$, for any $Y \in$ Top . $-\square Y$ and $[Y,-]$ are the partial functors of the bifunctors $-\square-$ : Top $\times$ Top $\rightarrow$ Top and $[-,-]:$ Top $\times$ Top $\rightarrow$ Top, if and only if $[-, S]:$ Top $^{\circ} \rightarrow$ Top defined by

$$
\begin{aligned}
& {[-, S](y)=[Y, S], Y \in \text { Top },} \\
& {[-, S](h)=[h, S]=\operatorname{hom}\left(h, I_{S}\right):[Y, S] \rightarrow\left[Y^{\prime}, S\right], h: Y^{\prime} \rightarrow Y,}
\end{aligned}
$$

is a contravariant functor.

In fact, by the universal property of initial topology and by the following commutative diagram 


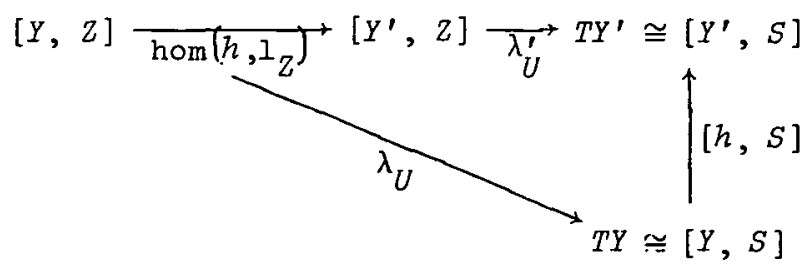

(where $h: Y^{\prime} \rightarrow Y, U \in O p Z$ ), each $[-, Z], Z \in$ Top is functorial. Since $[h, Z]=\operatorname{hom}\left(h, 1_{2}\right)$ is continuous, and the diagram

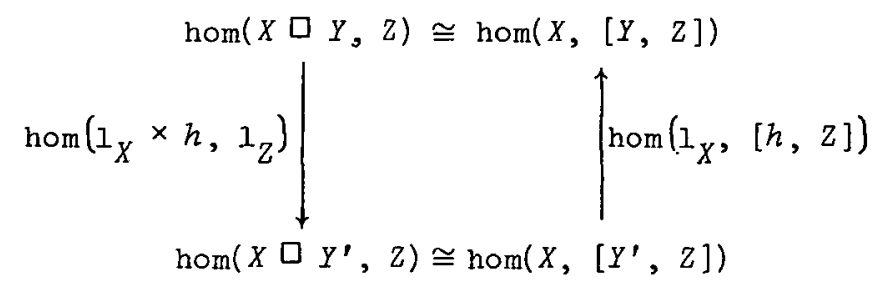

is commutative, then the function $1_{X} \times h: X \square Y^{\prime} \rightarrow X \square Y$ is continuous, and each $X \square-, X \in$ Top is a functor.

DEFINITION 1.5. A family of adjoining systems $(F(Y))_{Y \in T o p}$ is a functorial adjoining system if and only if $[-, S]:$ Top $\rightarrow$ Top is a functor.

For characterization of filters of a functorial adjoining system see wilker [11, p. 275-276].

2 .

In this section we give a characterization of those functorial adjoining systems that determine a monoidal closed structure (Top, $-\square_{-},[-,-],\{*\}$ ) on Top .

A topology on the set $O p Y, Y \in$ Top, is called a topological topology [8] if and only if it makes finite intersection and arbitrary union continuous operations.

LEMMA 2.1. If $F(Y)$ is an adjoining system for $Y$, then $T Y$ is a topological topology on $0 \mathrm{p} \mathrm{Y}$. Conversely, if $T Y$ is a topological topology on $\mathrm{Op} Y$, and $T Y$ has a subbase $F$, whose elements are filters, then $F$ is an adjoining system for $Y$.

Proof. If $F(Y)$ is an adjoining system, then $-\square Y \rightarrow[Y,-]$. 
Since $\{*\} \square Y \cong Y$, it follows by [10, p. 34, Theorem 4.1] or by [8], that $[Y, S] \cong T Y$ is a topological topology.

Conversely, the pair ( $Y, T Y$ ) with $T Y$ topological topology on Op $Y$, determines, again by [10, p. 34, Theorem 4.1], a pair of adjoint functors $H \dashv G$, where $H \cong-\square Y$ and $G \cong[Y,-]$. From Proposition $1.4, F$ must be an adjoining system on $0 p Y$.

Let $(F(Y))_{Y \in T o p}$ be a family of adjoining systems.

LEMMA 2.2. The exponential zow

$$
\psi_{X, Y, Z}:[X \square Y, Z] \rightarrow[X,[Y, Z]]
$$

is a homeomorphism, for any $X, Y, Z$ in Top, if and only if $\psi$ is a homeomorphism for any $X, Y$ in Top and $Z=S$.

Proof. Let $\psi_{X, Y, S}$ be a continuous map for any $X, Y \in$ Top . For the universal property of initial topology, to prove that $\psi_{X, Y, Z}$ is continuous, we have just to show that the composite

$$
[X \square Y, Z] \underset{\psi_{X, Y, Z}}{\longrightarrow}[X,[Y, Z]] \underset{\lambda_{V}}{\longrightarrow} T X
$$

is continuous for any $\lambda_{V}, V$ open set of $[Y, Z], Z \in$ Top . Let $W=W(F, U), F \in F(Y), U \in O p Z$ be a subbasic open set of $[Y, Z]$. It is easy to see that the characteristic map $\Phi_{W}$ coincides with the following composite map

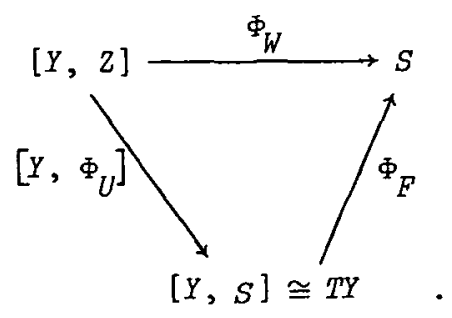

Let us consider now the diagram 


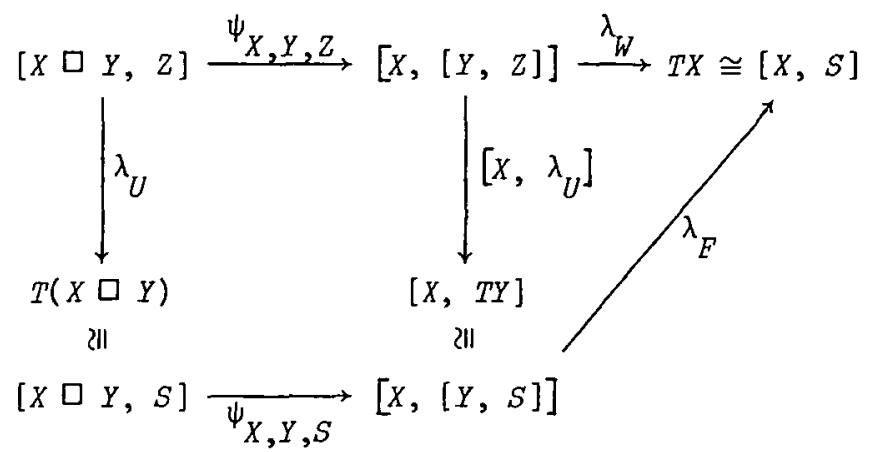

It is commutative for the naturality of $\psi$ and for (2.2.1), recalling that $\lambda_{W} \cong\left[X, \Phi_{W}\right], \lambda_{F} \cong\left[X, \Phi_{F}\right]$ and $\left[X, \lambda_{U}\right] \cong\left[X,\left[Y, \Phi_{U}\right]\right]$. It follows that $\lambda_{W} \cdot \psi_{X, Y, Z}$ is a continuous map, for any $X, Y, Z \in$ Top. If $\tilde{V}$ is a finite intersection of $W_{j}$, that is, $\tilde{V}=n W_{j}=n W\left(F_{j}, U_{j}\right)$ $(j=1, \ldots, n), F_{j} \in F(Y), y_{j} \in O \mathrm{p} \mathrm{Z}$, we can consider the diagram $(2.2 .2)$

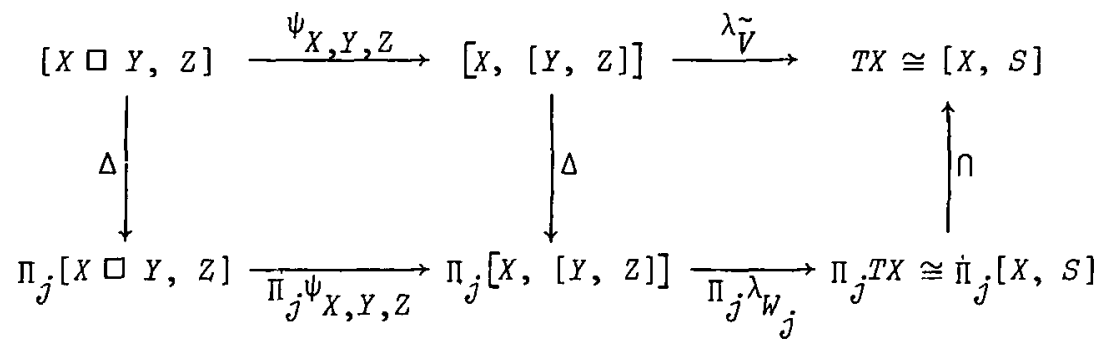

where $\Delta$ is the canonical diagonal map, and $\Pi$ denotes the topological product.

The commutativity of such a diagram follows from the naturality of $\Delta$ and the definition of $\tilde{V}$.

Since the diagonal map and the intersection map are continuous (Lemma 2.1), it follows that $\lambda_{V} \cdot \psi_{X, Y, Z}$ is continuous too, for any $X, Y, Z$ in Top .

Now, if $V$ is an arbitrary union of $\tilde{V}_{\alpha}, \tilde{V}_{\alpha}={ }_{j}{ }_{j}^{\alpha}$, $\left(j^{\alpha}: 1, \ldots, n^{\alpha}\right)$, we can prove the continuity of $\lambda_{V} \cdot \psi_{X, Y, Z}$ with a diagram similar to (2.2.2), where the intersection map is replaced with the union map. A similar proof applies to the inverse map $\psi_{X, Y, Z}^{-1}$, through 
the following commutative diagram:

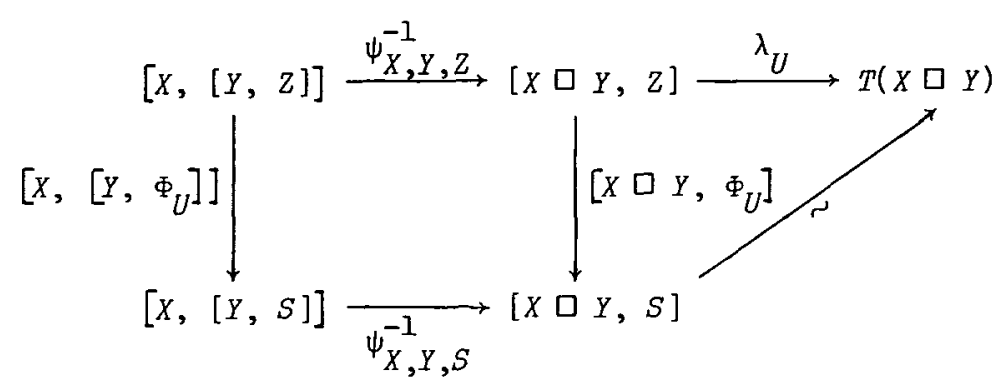

$U \in O p Z$.

PROPOSITION 2.3. A functorial adjoining system $(F(Y))_{Y \in T O P}$ determines a monoidal closed stmucture (Top, $-\square-,[-,-],\{*\}, r, 1, a)$ on Top if and only if

(i) $F(\{*\})$ induces on $0 \mathrm{p}\{*\}$ a topological topology $T(\{*\}) \cong S$;

(ii) $\psi_{X, Y, S}:[X \square Y, S] \cong[X,[Y, S]]$ is a homeomorphism for any $X, Y \in$ Top.

Proof. Any adjoining functorial system (see Definition 1.5) determines two bifunctors - - - : Top $\times$ Top $\rightarrow$ Top and $[-,-]: T_{0 p}{ }^{\circ} \times$ Top $\rightarrow$ Top such that, for any $Y \in T o p,-\square Y \rightarrow[Y,-]$.

Let $T(\{*\})$ be homeomorphic to a Sierpinski space, and let $\mu$ denote such homeomorphisms; we have necessarily $\mu(\{*\})=1$. Since $[\{t, X]$ is, up to bijection, the set $X$ with the initial topology with respect to the maps

$$
\lambda_{U}:[\{*\}, X] \rightarrow T(\{*\}) \cong S
$$

with $U \in O p X$, and since $\lambda_{U}^{-1}(\{*\}),\{*\} \in T(\{*\})$ is exactly the open set $U$, it follows that $[\{*\}, X] \cong X$, for any $X \in$ Top. We denote by $u_{X}$ such a natural homeomorphism, defined by $u_{X}\left(x_{0}\right)(*)=x_{0}, x_{0} \epsilon X$. Then, from Lemma 2.2 and from [6, p. 495, Theorem 5.10], it follows that (Top, $-\square-,[-,-],\{*\}, r, 1, a$ ) is a monoidal closed structure on Top , where $r, 1, a$ are enrichments in Top of the canonical natural isomorphisms of Set. 
Suppose now we use the same symbol both for an open set of $Y$ and for its characteristic associated map.

The space $[X,[Y, S]]$ has the initial topology with respect to the maps

$$
\lambda_{U}: \operatorname{hom}(X,[Y, S]) \rightarrow T X
$$

with $\quad U \in T Y$.

If $\psi:[X \square Y, S] \cong[X,[Y, S]]$, it follows that the family

$\psi_{X, Y, S}^{-1}\left(\lambda_{U}^{-1}(V)\right), U \in T Y$ and $V \in T X$, is an open subbase of $[X \square Y, S]$; furthermore for properties of initial topology (see also diagram (2.2.2)), it suffices to consider $U \in F(Y), V \in F(X)$.

Let us denote by $H(F, G), F \in F(Y), G \in F(X)$, the following subset of $0 p(X \square Y) \quad$ that is, up to bijection, just the set $\left.\psi_{X, Y, S}^{-1}\left(\lambda_{F}^{-1}(G)\right)\right\}$

$$
H(F, G)=\left\{A, A \in O p(X \square Y) \mid Q_{A}=\left\{x, x \in X \mid A_{x} \in F\right\} \in G\right\} .
$$

PROPOSITION 2.4. If $F(Y)$ and $F(X)$ are adjoining systems on $0 p Y$ and $O p X$ respectively, then the family $\{H(F, G) \mid F \in F(Y), G \in F(X)\}$ is an adjoining system on op $X \square Y$.

Proof. It is easily seen that $H(F, G)$ is a filter on $0 p \times \square Y$, for any $F \in F(Y)$ and $G \in F(X)$. To prove that $H(F, G)$ is a compact filter, suppose that $\mathrm{U} A_{i} \in H(F, G), i \in I$ and $A_{i} \in$ Op X $\square$.

If $Q=\left\{x, x \in X \mid \cup\left(A_{i}\right)_{x} \in F, i \in I\right\}$, then, by definition of $H(F, G)$, it follows that $Q \in G$.

Since $F$ is a compact filter, for any $\bar{x} \in Q$ there is a finite subset $J_{\bar{x}}$ of $I$ such that $U\left(A_{i}\right]_{\bar{x}} \in F, i \in J_{\bar{x}}$.

If $Q_{\bar{x}}=\left\{x, x \in X \mid \cup\left(A_{i}\right)_{x} \in F, i \in J_{\bar{x}}\right\}$, then $Q=\cup Q_{\bar{x}}, \bar{x} \in Q$. Since $G$ is a compact filter, it follows that there is a finite subset $L$ of $Q$ such that $U_{\bar{x}} \in G, \bar{x} \in L$. If we define $K=U_{J_{\bar{x}}}, \bar{x} \in L$, we obtain a finite subset of $I$ and $\mathrm{U} A_{i} \in H(F, G)$ with $i \in K$.

Now we are going to prove that the family 


$$
\{H(F, G) \mid F \in F(Y), G \in F(X)\}
$$

verifies condition ( $i$ ) of the definition of adjoining systems. If $A_{1} \cup A_{2} \in H(F, G)$, with $A_{1}, A_{2} \in O \mathrm{p} X \square Y$, let us denote by $Q$ the set $\left\{x, x \in X \mid\left(A_{1}\right)_{x} \cup\left(A_{2}\right)_{x} \in F\right\} ;$ then $Q \in G$.

Since $F$ is an adjoining system it follows that, for any $\bar{x} \in Q$, $\left(A_{1}\right)_{\bar{x}} \in\left(F_{1}\right)_{\bar{x}}$ and $\left(A_{2}\right)_{\bar{x}} \in\left(F_{2}\right)_{\bar{x}}$ where $\left(F_{1}\right)_{\bar{x}}$ and $\left(F_{2}\right)_{\bar{x}}$ are finite intersections of members of $F(Y)$ and $\left(F_{1}\right)_{\bar{x}} \cap\left(F_{2}\right)_{\bar{x}} \subseteq F$.

If we define

$$
P_{\bar{x}}=\left\{x, x \in X \mid\left(A_{1}\right)_{x} \in\left(F_{1}\right)_{\bar{x}}\right\}
$$

and

$$
R_{\bar{x}}=\left\{x, x \in X \mid\left(A_{2}\right)_{x} \in\left(F_{2}\right)_{-}\right\},
$$

then it is easy to see that $Q=U\left(P_{\bar{x}} \cap R_{\bar{x}}\right), \bar{x} \in Q$.

Since $G$ is a compact filter, it follows that there is a finite subset $K$ of $Q$ such that $U\left(P_{\bar{x}} \cap R_{\bar{x}}\right) \in G, \bar{x} \in K$. As the general finite case follows by induction, only two elements $x_{1}$ and $x_{2}$ will suffice. From $\left(P_{x_{1}} \cap R_{x_{1}}\right) \cup\left(P_{x_{2}} \cap R_{x_{2}}\right) \in G$, and property (ii) of the adjoining system $G$, it follows that $P_{x_{1}} \cap R_{x_{1}} \in G_{1}$ and $P_{x_{2}} \cap R_{x_{2}} \in G_{2}$, where $G_{1}$ and $G_{2}$ are finite intersections of filters belonging to $F(X)$ and $G_{1} \cap G_{2} \subseteq G$.

Recall that, if $F$ is a finite intersection of $F_{\alpha}$, with $F_{\alpha} \in F(Y)$ and $G$ is a finite intersection of $G_{B}$ with $G_{B} \in F(X)$, then $H(F, G)=\cap H\left(F_{\alpha}, G_{\beta}\right)$.

Consider now the filters

$$
H\left(\left(F_{j}\right)_{x_{i}}, G_{i}\right), i=1,2, j=1,2 ;
$$

it follows that $A_{1} \in H\left(\left(F_{1}\right)_{x_{1}}, G_{1}\right) \cap H\left(\left(F_{1}\right)_{x_{2}}, G_{2}\right)$ and 
$A_{2} \in H\left(\left(F_{2}\right)_{x_{1}}, G_{1}\right) \cap H\left(\left(F_{2}\right)_{x_{2}}, G_{2}\right)$. To verify that

$$
\cap H\left(\left(F_{j}\right)_{x_{i}}, G_{i}\right) \subseteq H(F, G), i=1,2, j=1,2 \text {, }
$$

let $A$ be an open set of $X \square Y$ and $A \in \cap H\left(\left(F_{j}\right)_{x_{i}}, G_{i}\right)$. If $L$ denotes the set $\left\{x, x \in X \mid A_{x} \in F\right\}$, we must prove that $L \in G$.

Let us define the sets

$$
L_{j, i}=\left\{x, x \in X \mid A_{x} \in\left(F_{j}\right)_{x_{i}}\right\}, i=1,2, j=1,2 ;
$$

it follows that $L_{j, i} \in G_{i}$ and $L_{1, i} \cap L_{2, i} \subseteq G_{i}, i=1,2$. Furthermore, if we define $L^{*}=U\left(L_{1, i} \cap L_{2, i}\right), i=1,2$, then $L^{*} \in G_{1} \cap G_{2}$, hence $L^{*} \in G$.

It suffices now to prove that $L^{*} \subseteq L$. If $x \in L^{*}$, then $x \in L_{1,1} \cap L_{2,1}$ or $x \in L_{1,2} \cap L_{2,2}$. Suppose $x \in L_{1,1} \cap L_{2,1}$; then, since $A_{x} \in\left(F_{1}\right)_{x_{1}} \cap\left(F_{2}\right)_{x_{1}}$, it follows $A_{x} \in F$ and $x \in L$.

The topological space on $0 p X \square Y$, having the family $\{H(F, G), F \in F(Y), G \in F(X)\}$, as an open subbase, will be denoted by $\langle X, Y\rangle$. Then condition (ii) of Proposition 2.3 can be replaced by

$$
\text { (ii)' } T(X \square Y) \cong(X, Y)
$$

for any $X, Y \in$ Top.

It is interesting to observe that it is possible to give a characterization of all monoidal closed stmuctures on Top, generalizing Proposition 2.3 to the case of arbitrary topological topology TY (not given by filters), and recalling the theorem of Isbell on the characterization of adjoint functors in Top [8].

3.

For any family $F(Y)$ of filters on $O p Y$, let us denote by $F^{\prime}(Y)$ the system of all filters which can be written as finite intersection of filters belonging to $F(Y)$. Of course $F(Y)$ is an adjoining system if and only if $F^{\prime}(Y)$ is an adjoining system. 
All the examples of monoidal closed structures on Top, given by Booth and $\mathrm{Tillotson}$ in [2], and by Greve in [7] are determined by filters. In such examples, if $A$ is the opportune class of spaces used to define the structure, then the associated functorial adjoining system is given by families of generated filters of the form

$$
\{\langle f(A)\rangle \mid A \in A, f: A \rightarrow Y\}_{Y \in T o p} .
$$

In particular, the canonical symmetric monoidal closed structure (topology of separate continuity on $X \times Y$, and pointwise convergence on $\operatorname{hom}(Y, Z))$ is determined by the functorial adjoining system $\{\langle y\rangle \mid y \in Y\}_{Y \in T o p}$ of all principal ultrafilters of open sets. Moreover $\{\langle y\rangle \mid y \in Y\}^{\prime}=E(Y)$, where $E(Y)$ is the minimal adjoining system of [11].

Consider now the trivial functorial adjoining system $J(J(Y)=\varnothing$, for any $Y \in$ Top) and the maximal functorial adjoining system $M$ of [11]. Condition (i) of Proposition 2.3 is satisfied by $M$, but not by $J$. Condition ( $i i$ ) is satisfied by $J$; as for $M$, the only result we can get is, by Proposition 2.4, $T(X \square Y) \geq\langle X, Y\rangle$, for any $X, Y \in$ Top .

If we assume all spaces to be Hausdorff, then $M$ determines a monoidal structure (Haus, $-x_{S}-,[-,-]_{c o},\{*\}$ ), where co denotes the compact-open topology ([4]). To prove this result it suffices to recall that each compact filter $F$ on $0 p Y, Y \in$ Haus, is compactly generated [8, p. 331]; then, by [11, p. 278, Theorem 7], it follows that the topological space on $\operatorname{hom}(Y, Z)$ determined by $M$ coincides with $[Y, Z]_{\text {co }}$, for any $Y, Z \in$ Top.

\section{References}

[1] Richard Arens and James Dugundji, "Topologies for function spaces", Pacific J. Math. 1 (1951), 5-31.

[2] P. Booth and J. Tillotson, "Monoidal closed, cartesian closed and convenient categories of topological spaces", Pacific J. Math. $88(1980), 35-53$. 
[3] R. Brown, "Ten topologies for $X \times Y$ ", Quart. J. Math. Oxford (2) 14 (1963), 303-319.

[4] R. Brown, "Function spaces and product topologies", Quart. J. Math. Oxford (2) 15 (1964), 238-250.

[5] Juraj Cinčura, "Tensor products in the category of topological spaces", Comment. Math. Univ. Carolin. 20 (1979), 431-446.

[6] Samuel Eilenberg, G. Max Kelly, "Closed categories", Proc. Conf. Categorical AZgebra, La Jolla, 1965, 421-562 (Springer-Verlag, Berlin, Heidelberg, New York, 1966).

[7] Georg Greve, "How many monoidal closed structures are there in Top ?", Arch. Math. (Basel) 34 (1980), 538-539.

[8] John R. Isbell, "Function spaces and adjoints", Math. Scand. 36 (1975), 317-339.

[9] Maria Cristina Pedicchio e Fabio Rossi, A remark on monoidal closed structures on Top (Quaderni Matematici, II Serie, 55. Istituto di Matematica, Universita' Degli Studi di Trieste, 1982).

[10] Maria Cristina Vipera, Endofuntomi aggiunti di Top (Quaderno del gruppo di ricerca Algebra - Geometria - Topologia, 4. Dipartimento di Matematica, dell'Universita di Perugia, 1980).

[11] Peter Wilker, "Adjoint product and hom functors in general topology", Pacific J. Math. 34 (1970), 269-283.

\footnotetext{
Istituto Matematica,

Universita di Trieste,

Piazzale Europa I,

Trieste,

italy.
} 